\title{
PLCD3, a flotillin2-interacting protein, is involved in proliferation, migration and invasion of nasopharyngeal carcinoma cells
}

\author{
WEIDONG LIU ${ }^{1,2^{*}}$, XUXU LIU $^{1,2^{*}}$, LEI WANG $^{1,2}$, BIN ZHU $^{1,2}$, CHANG ZHANG $^{1,2}$, WEI JIA ${ }^{1,2}$, HECHENG ZHU $^{3}$, \\ XINGDONG LIU ${ }^{3}$, MEIZUO ZHONG $^{3}$, DAN XIE $^{4}$, YANYU LIU $^{1,2}$, SHASHA LI $^{1,2}$, JIA SHI $^{1,2}$, \\ JIANXING LIN $^{1,2}$, XIAOMENG XIA ${ }^{5}$, XINGJUN JIANG ${ }^{6}$ and CAIPING REN ${ }^{1,2}$
}

\author{
${ }^{1}$ The Key Laboratory of Carcinogenesis of the Chinese Ministry of Health and the Key Laboratory of Carcinogenesis and \\ Cancer Invasion of the Chinese Ministry of Education, Xiangya Hospital, Central South University, Changsha, Hunan 410008; \\ ${ }^{2}$ Cancer Research Institute, Collaborative Innovation Center for Cancer Medicine, School of Basic Medical Science, \\ Central South University, Changsha, Hunan 410078; ${ }^{3}$ Changsha Kexin Cancer Hospital, Changsha, Hunan 410205; \\ ${ }^{4}$ State Key Laboratory of Oncology in South China, Collaborative Innovation Center for Cancer Medicine, \\ Sun Yat-sen University Cancer Center, Guangzhou, Hunan 510060; ${ }^{5}$ Department of Gynecology and Obstetrics, \\ The Second Xiangya Hospital, Central South University, Changsha, Hunan 410011; \\ ${ }^{6}$ Department of Neurosurgery, Xiangya Hospital, Central South University, \\ Changsha, Hunan 410008, P.R. China
}

Received April 1, 2017; Accepted September 18, 2017

DOI: $10.3892 /$ or.2017.6080

\begin{abstract}
Phospholipase C (PLC) is a pivotal enzyme in the phosphoinositide pathway that promotes the second messengers, diacylglycerol (DAG) and inositol 1,4,5-trisphosphate (IP3), to participate in eukaryotic signal transduction. Several PLC isozymes are associated with cancer, such as PLC- $\beta 1$, PLC- $\delta 1$, PLC- $\varepsilon$ and PLC- $\gamma 1$. However, the role of PLC- $\delta 3$ (PLCD3) in nasopharyngeal carcinoma (NPC) has not been investigated to date. In our previous study, we demonstrated that flotillin2 (Flot2) plays a pro-neoplastic role in NPC and is involved in tumour progression and metastasis. In the present study, we screened the interacting proteins of Flot2 using the yeast two-hybrid $(\mathrm{Y} 2 \mathrm{H})$ method and verified the interaction between PLCD3 and Flot2 by co-immunoprecipitation. We also investigated the biological functions of PLCD3 in NPC. Inhibition of PLCD3 expression impaired the malignant poten-
\end{abstract}

Correspondence to: Professor Caiping Ren, The Key Laboratory of Carcinogenesis of the Chinese Ministry of Health and the Key Laboratory of Carcinogenesis and Cancer Invasion of the Chinese Ministry of Education, Xiangya Hospital, Central South University, Changsha, Hunan 410008, P.R. China

E-mail: rencaiping@csu.edu.cn

Professor Xingjun Jiang, Department of Neurosurgery, Xiangya Hospital, Central South University, Changsha, Hunan 410008, P.R. China

E-mail: jiangxj@csu.edu.cn

*Contributed equally

Key words: flotllin2, interaction protein, PLCD3, yeast two-hybrid, nasopharyngeal carcinoma cells, tumour progression, metastasis tial of 5-8F, a highly metastatic NPC cell line, by restraining its growth, proliferation, mobility and migration. The present study demonstrated that PLCD3 may be an oncogenic protein in NPC and that it plays an important role in the progression of NPC partially by interacting with Flot2.

\section{Introduction}

Nasopharyngeal carcinoma (NPC), which is a form of squamous cell carcinoma (1), is a head and neck malignant tumour. NPC is endemic in southeast Asia and southern areas of China (2). For the treatment of NPC, radiotherapy can be used alone or in combination with chemotherapy and surgery (3). NPC is characterised by early cervical lymph node and distant metastases. The probabilities of treatment failure and relapse in NPC patients with distant metastasis have not been significantly reduced to date. Therefore, comprehensive studies on the causes and biological processes of NPC metastasis are needed to improve the therapeutic efficacy for this malignancy.

Yeast two-hybrid $(\mathrm{Y} 2 \mathrm{H})$ is a method to screen unknown proteins that interact with known proteins $(4,5)$. In our previous study, we discovered that flotillin2 (Flot2) can promote the progression and metastasis of NPC and revealed the molecular mechanisms by which it exerts this function (6-8). In the present study, we aimed to identify the proteins that interact with Flot 2 in NPC using a $\mathrm{Y} 2 \mathrm{H}$ system. Among the five obtained proteins, PLCD3 attracted our attention since it is located on the cell membrane similar to Flot2.

Flot2 is a lipid raft marker protein, and directly interacts with signaling molecules such as receptors, kinases, adhesion molecules and $\mathrm{G}$ proteins. It also serves as a tumour regulator by regulating cell proliferation, differentiation, apoptosis, adhesion and invasion. It has been reported that Flot 2 participates in the development of several types of malignant tumours 
such as breast cancer, melanoma, gastric and cervical cancer, and NPC (9).

PLCD3 is a member of the phospholipase C (PLC) family. PLC is a pivotal enzyme in the phosphoinositide pathway and is involved in eukaryotic signal transduction through the generation of two second messengers: diacylglycerol (DAG) and inositol 1,4,5-trisphosphate (IP3). The former mediates the activation of protein kinase $\mathrm{C}$ (PKC) (10), and the latter regulates the release of $\mathrm{Ca}^{2+}$ from intracellular stores $(11,12)$. Since the 1950s, 13 isozymes of six PLC family members in mammals have been reported, including PLC $\beta 1-4$, PLC $\gamma 1-2$, PLC81-4, PLC- $\varepsilon$, PLC- $\zeta$ and PLC- $\eta$ (13-16). The core structures of these proteins include a $\mathrm{PH}$ domain in $\mathrm{N}$-terminus, a catalytic centre formed by $\mathrm{X}$ and $\mathrm{Y}$ regions, an EF-hands motif and a C2 domain in the C-terminal region (17). PLC isozymes have both common and characteristic features that reflect their physiological and pathological functions, so each isozyme may be associated with specific human disease (18). PLC- $\beta 1$ is involved in schizophrenia $(19,20)$, myelodysplastic syndromes (MDS) (21-23), leukemogenesis (24) and diabetes (25). PLC- $\gamma 1$ is implicated in epilepsy (26), cancer cell invasion and metastasis in brain tumours (27) and breast tumour formation (28). PLC- $\delta 1$ is associated with obesity (29), neurodegenerative disorders (30), and Alzheimer disease (31) and may be a tumour suppressor $(32,33)$. PLC- $\varepsilon$ may function as a tumour suppressor $(34,35)$. PLC- $\zeta$ is related to male infertility (36), whereas PLC- $\beta 3$ is associated with lymphomas and other tumours (37).

In the present study, we identified a Flot2-interacting protein, PLCD3, which may play an important role in NPC progression.

\section{Materials and methods}

Cell culture. Briefly, 5-8F NPC cells were maintained by our laboratory and grown in RPMI-1640 media (Invitrogen, Carlsbad, CA, USA) supplemented with $10 \%$ fetal bovine serum (FBS) in a humidified atmosphere with $5 \% \mathrm{CO}_{2}$ at $37^{\circ} \mathrm{C}$.

Yeast two-hybrid. The culture conditions and treatment methods of yeast strains Y2Gold and Y187 were similar to those described previously (38). PMD18-T vector (kept by our laboratory) was ligated to the open reading frame (ORF) of Flot2, which was amplified by PCR using a sense primer (5'-CGAATTCATGAGTAGCTCCCACTCTC-3') and an antisense primer (5'-CGGATCCTCAGGGCACCACAACGC-3') from 5-8F cDNA. Then, the yielded product and pGBKT7-BD were double digested by EcoRI and BamHI (both from Takara, Shiga, Japan), respectively. The digested DNA fragment was recombined in vitro. The obtained yeast bait vector pGBKT7Flot2 was transfected into yeast bait strain Y2Gold.

The cDNA of 5-8F was reverse transcribed using SMART ${ }^{\mathrm{TM}}$ technology and co-transfected into yeast library strain Y187 (both from Clontech, Shiga, Japan) with pGADT7-Rec vector, which was then plated on SD/-leu agar medium. After 4-5 days, the colonies were pooled in rich broth medium, divided into $1 \mathrm{ml}$-aliquots and cryopreserved. The cDNA library screening was performed according to our previous protocol (38).

Co-immunoprecipitation (Co-IP). The ORF of Flot2 was cloned into a BamHI- and NotI-digested pEF1/myc-His B vector (Invitrogen), which is a 6.2-kb expression vector used to overexpress recombinant protein. pFlag-CMV-3 (Sigma, Rockford, IL, USA) is a 6.2-kb expression vector transiently or stably expressed in mammals, in which the N-terminal Flag can form fusion protein with a correctly inserted ORF. The ORF of PLCD3 was amplified from 5-8F cDNA by PCR using the sense primer (5'-CAAGCTTATGCTGTGCGGCCGC TGGA-3') and the antisense primer (5'-CGGATCCTCAGGA GCGCTGGATGCGGAT-3'), and then subcloned into pFlagCMV-3. Then, pEF1/myc-His-Flot2 and pFlag-CMV-PLCD3 were transfected into $293 \mathrm{~T}$ cells with Lipofectamine $2000^{\mathrm{TM}}$ (Invitrogen). All experiments were performed according to a previously published protocol (38). Anti-Flag (Sigma) was used for immunoprecipitation. Anti-His (ProteinTech, Wuhan, China) was used for immunoblotting.

siRNA treatment. Cells were transfected with RNAiMax (Invitrogen) and PLCD3 siRNA or control siRNA was synthesised by RiboBio Co. (Guangzhou, China). The siRNA complex was formed in Opti I medium (Invitrogen) following the manufacturer's recommendations. The optimal concentrations tested to effectively reduce expression of PLCD3 $24 \mathrm{~h}$ after seeding $2 \times 10^{5}$ cells in $60-\mathrm{mm}$ dishes were $7.5 \mathrm{pmol}$ siRNA and $5 \mu \mathrm{l}$ of RNAiMax/1 ml of growth medium. Human PLCD3 siRNA and control siRNA were purchased from RiboBio Co. The siRNA sequences are as follows: si-1, GGATGAACT CAGCCAACT; si-2, GCCCACT ACTTCATCTCTT; and si-3, GGAGCCCGTCATCTATCAT. In our experiments, si-1 and si-2 were most effective on suppressing PLCD3 expression.

Real-time $q P C R$. Real-time qPCR was performed as previously described $(39,40)$. Briefly, $48 \mathrm{~h}$ after treatment, each $60-\mathrm{mm}$ dish of monolayer cells was washed twice with $2 \mathrm{ml} \mathrm{D-Hank's} \mathrm{solution.} \mathrm{Then,} \mathrm{total} \mathrm{RNA} \mathrm{was} \mathrm{isolated.}$ qPCR reactions were performed as previously described. Glyceraldehyde-3-phosphate dehydrogenase (GAPDH) was used as an internal control. PLCD3 was amplified using the forward primer (5'-CAAGCTTATGCTGTGCGGCCGCT GGA-3') and the reverse primer (5'-CGGATCCTCAGGAG CGCTGGATGCGGAT-3'). This experiment was independently repeated three times.

Western blot analysis. Western blotting was performed according to a previous protocol (2). Rabbit polyclonal anti-PLCD3, anti-MMP2, anti-MMP9, anti-Snai1, anti-Ecadherin, anti-vimentin, anti-N-cadherin and anti-Flot2 were purchased from ProteinTech. Mouse anti- $\beta$-actin was purchased from Sigma. Rabbit anti-mouse IgG was purchased from Abcam (1:40,000; Cambridge, MA, USA). Quantification of signal intensity [integral optical density (IOD)] was performed with Gel-Pro Analyzer software (version 4.0). Expression changes in protein levels were assessed by IOD, and the intensity was normalised to the $\beta$-actin signal. This experiment was independently repeated three times.

Colony formation assay. Twenty-four hours after treatment, the transfected cells were digested with trypsin and seeded in $60-\mathrm{mm}$ dishes at 1,000 cells/well. Colony formation assays 

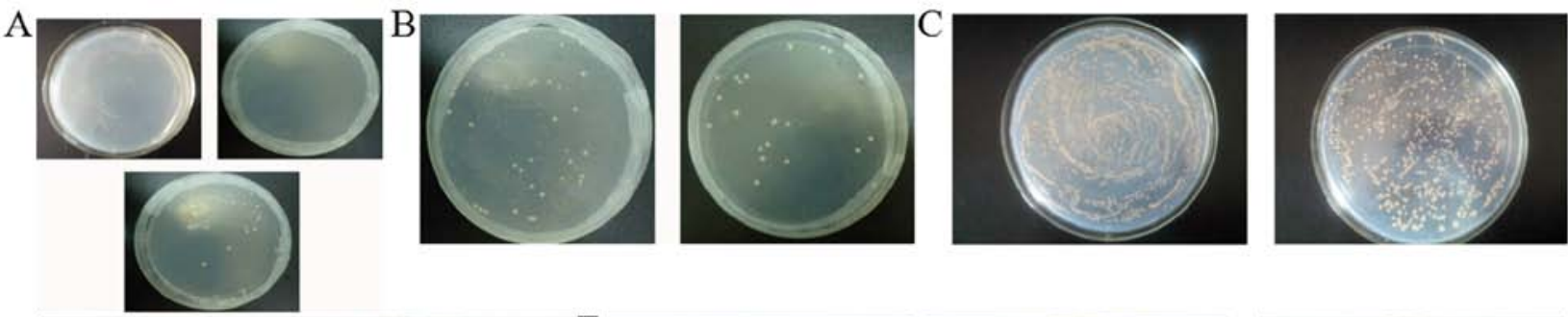

$\mathrm{D}$
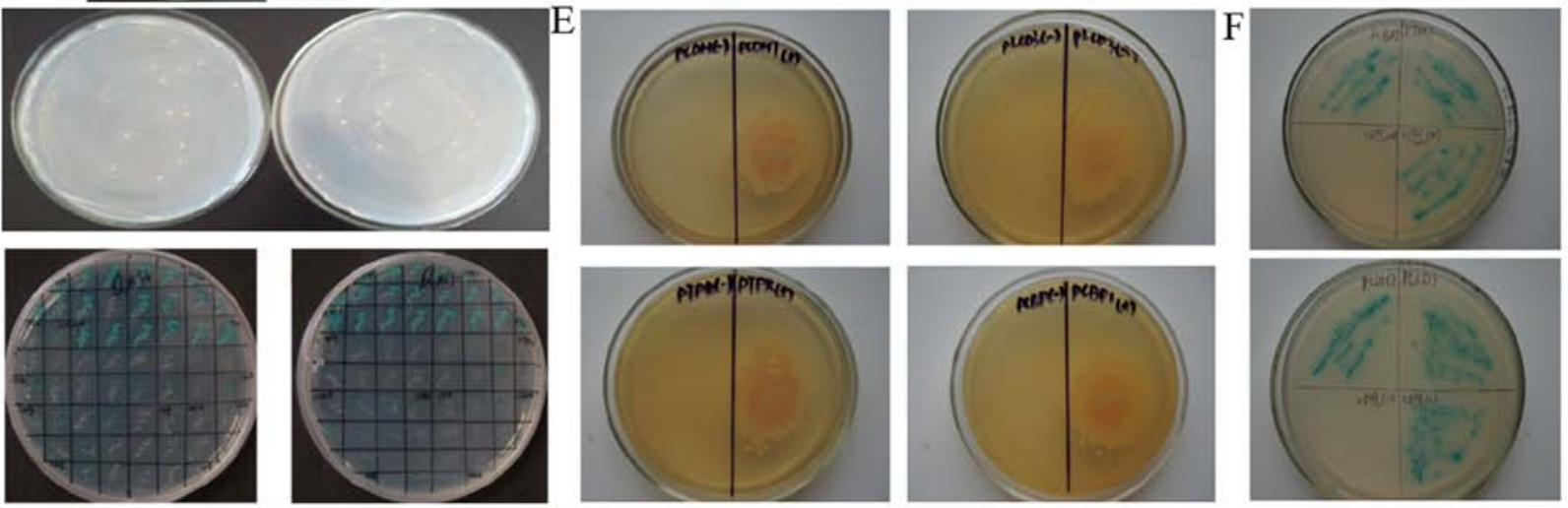

Figure 1. Detection of the autonomous activation and toxicity of the Y2H system and screening of the interaction proteins of Flot2. (A) No autonomous activation was detected since Y2H gold yeast transfected with pGBKT7-Flot 2 was able to proliferate on SD/-Trp/X- $\alpha$-Gal (below), but not on SD/-His/-Trp/X- $\alpha$-Gal (upper left) or SD/-Ade/-Trp/X- $\alpha$-Gal (upper right). (B) No toxicity was detected given no obvious differences in growth rate and colony size between the pGBKT7-Flot2-Y2Hgold (left panel) and the pGBKT7-BD-Y2Hgold group (right panel). (C) The quality of the 5-8F cDNA library was verified by calculating the titre of the library (left panel 1:1; right panel 1:100). (D) Sixty-five positive clones were obtained on QDO plates (upper panel), and 32 clones exhibited blue colour (lower panel). (E and F) Yeast co-transfected with the pGBKT7-Flot2 vector and any of the four pGADT7-candidate vectors was able to grow on QDO plates (E), and further proliferate and appear blue when transferred to SD/-Ade/-His/-Leu/-Trp/ABA/X- $\alpha$-Gal plates. (F) Yeast transfected with pGBKT7-BD and pGADT7-candidate vectors were not able to grow on QDO plates.

were performed according to a published manual (41). The data are expressed as the means \pm SD of colony numbers. This experiment was independently repeated three times.

In vitro cell proliferation assay. Twenty-four hours after treatment, the transfected cells were digested with trypsin and seeded in 96-well dishes at 3,000 cells/well. MTT assays were performed to assess the effect of PLCD3 on 5-8F proliferation in accordance with a published protocol (42). The experiment was independently repeated three times.

Fluorescence-activated cell sorting (FACS). Twenty-four to forty-eight hours after treatment, the transfected cells were digested with trypsin, and then, FACS analysis was performed as previously described (43). The experiment was independently repeated three times.

Matrigel invasion assay. Twenty-four hours after treatment, Matrigel invasion assays were performed according to a published protocol (8). The experiment was independently repeated three times.

Wound healing assay. Forty-eight hours after treatment, wound healing assays were performed according to a previous protocol (43). The experiment was independently repeated three times.

Statistical analysis. Statistical analyses were executed using SPSS version 18.0 statistical software (SPSS, Inc., Chicago, IL, USA). Differences were analysed using Student's t-test. A $\mathrm{P}$-value $\leq 0.05$ was indicative of statistical significance.

\section{Results}

No autonomous activity and toxicity of the pGBKT7Flot2 yeast bait vector. Y2Hgold yeast cells transfected with pGBKT7-Flot2 were plated on SD/-Trp/X- $\alpha$-Gal, $\mathrm{SD} /$-His/-Trp/X- $\alpha-\mathrm{Gal}$ or SD/-Ade/-Trp/X- $\alpha-\mathrm{Gal}$ for 3-5 days. pGBKT7-Flot2-Y2Hgold yeast can proliferate on $\mathrm{SD} /-\operatorname{Trp} / \mathrm{X}-\alpha-$ Gal (Fig. 1A, below), but not on SD/-His/-Trp/X$\alpha$-Gal (Fig. 1A, upper left) or SD/-Ade/-Trp/X- $\alpha$-Gal (Fig. 1A, upper right), suggesting the lack of autonomous activity of the yeast bait vector in the tested yeast. Y2Hgold yeast transfected with pGBKT7-Flot2 or pGBKT7-BD were individually plated on SD/-Trp plates. No obvious differences in the growth rate and colony size were noted between these two groups (Fig. 1B). The results above demonstrate that the $\mathrm{Y} 2 \mathrm{H}$ system could be used in the following experiments.

Four candidate Flot2-interacting proteins are identified. The quality of the 5-8F cDNA library was verified by calculating the titre of the library (Fig. 1C). In total, 65 positive candidate clones were observed on QDO (SD/-Ade/-His/-Leu/-Trp) plates (Fig. 1D, upper panel), and 32 positive clones with blue colour appeared on SD/-Ade/-His/-Leu/-Trp/ABA/X- $\alpha-\mathrm{Gal}$ plates (Fig. 1D, lower panel). The positive candidate clones were amplified. The plasmids were extracted, and the target cDNAs were sequenced. Finally, five candidate Flot2-interacting proteins were obtained (Table I), including Copine-II PCDH7, PCBP1, PTPRJ and PLCD3. After further co-transfection experiments, only PCDH7, PCBP1, PTPRJ and PLCD3 proteins were confirmed to interact with Flot2. Only yeasts 
Table. I. BLAST results of positive clones in the Yeast two-hybrid analysis.

\begin{tabular}{lllcr}
\hline Protein no. & \multicolumn{1}{c}{ Protein name } & Gene & NCBI protein accession no. & Max identify (\%) \\
\hline 1 & CPNE2 & Copine-II & XP_003823796 & 99 \\
2 & Protocadherin-7 & PCDH7 & XP_006184446 & 99 \\
3 & Poly(C)-binding protein 1 & PCBP1 & XP_003922705 & 100 \\
4 & PTPRJ protein & PTPRJ & AAH63417 & 100 \\
5 & PLCD3 protein, partial & PLCD3 & AAH10668 & 99 \\
\hline
\end{tabular}

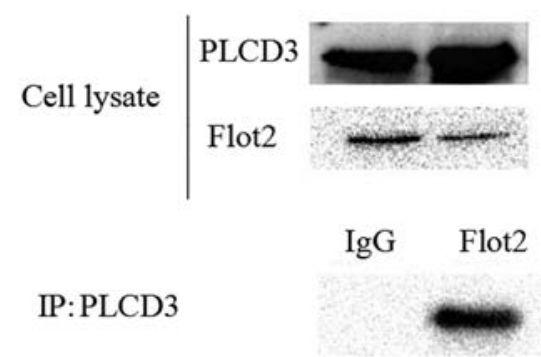

Figure 2. Identification of the interaction between Flot 2 and PLCD3 by coimmunoprecipitation (Co-IP). pFlag-PLCD3 and pHis-Flot2 vectors were co-transfected into $293 \mathrm{~T}$ cells. Thirty-six hours after transfection, the lysates were incubated with the anti-Flag antibody, and then detected using the antiHis antibody.
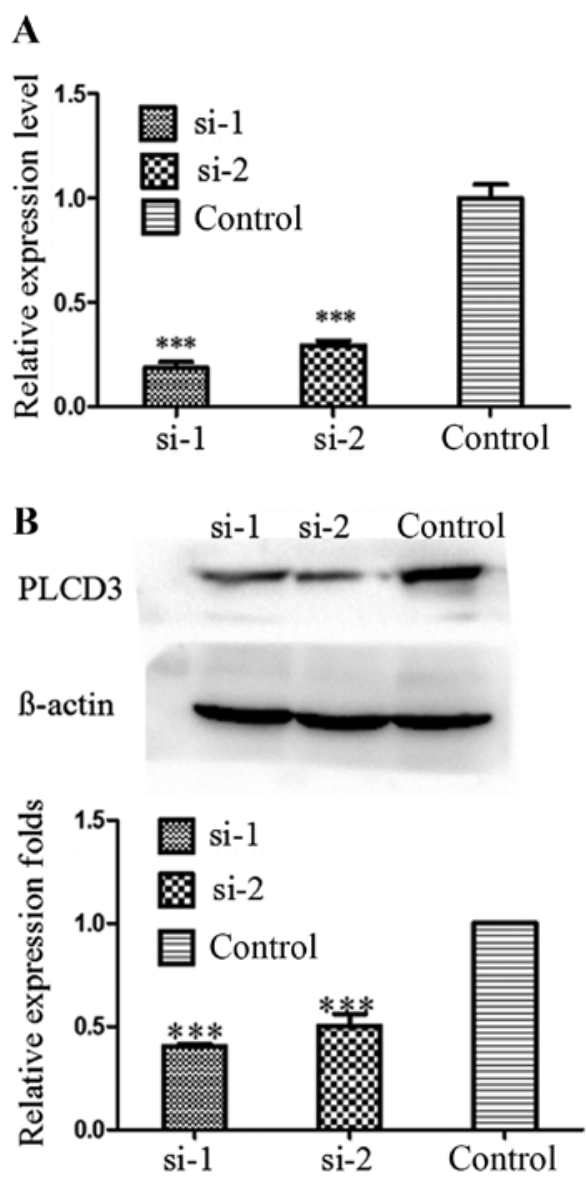

Figure 3. Knockdown of PLCD3 in 5-8F cells by siRNA. (A) qPCR detected reduced PLCD3 mRNA levels in 5-8F cells after transfection with PLCD3 siRNA. (B) Western blot analysis detected reduced PLCD3 protein level in $5-8 \mathrm{~F}$ cells after transfection with siRNA; ${ }^{* * *} \mathrm{P}<0.001$ compared with the control group.

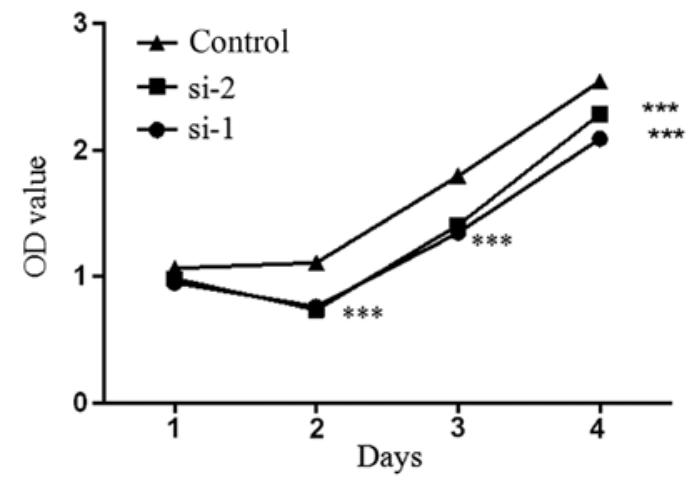

Figure 4. Effect of PLCD3 downregulation on 5-8F cell proliferation. Growth curves of 5-8F cells were assessed after transfection with PLCD3 siRNA or control scramble RNA. The optical density (OD) at $595 \mathrm{~nm}$ was measured at the same time every day; ${ }^{* * *} \mathrm{P}<0.001$ compared with the control group.

transfected with pGBKT7-Flot2 and pGADT7-candidate vectors encoding PCDH7, PCBP1, PTPRJ and PLCD3 was able to grow on $\mathrm{QDO}$ plates, and further proliferate and appear blue when grown on SD/-Ade/-His/-Leu/-Trp/ABA/X- $\alpha$-Gal plates, whereas yeasts transfected with pGBKT7-BD and pGADT7Copine-II vectors could not (Fig. $1 \mathrm{E}$ and F), indicating that these four candidate proteins interact with Flot2.

Interaction between Flot2 and PLCD3 is further confirmed by Co-IP. To further verify the interaction between Flot 2 and PLCD3, a mammal expression plasmid encoding His-Flot2 (ORF) and a plasmid encoding Flag-PLCD3 were co-transfected into HEK-293T cells. The lysates were immunoprecipitated with anti-Flag antibody, and then immunoblotted with an antiHis antibody. The His tag could be detected (Fig. 2), which confirmed the interaction between PLCD3 and Flot2.

PLCD3 expression is silenced in 5-8F cells by siRNA transfection. To verify the knockdown efficiency after transfection with PLCD3 siRNAs (si-1 or si-2), total RNA and protein of $5-8 \mathrm{~F}$ cells were extracted at 24 and $48 \mathrm{~h}$ after treatment, separately. Then, qPCR and western blotting were performed. As shown in Fig. 3A and B, the knockdown efficiency of PLCD3 mRNA was $\sim 80 \%$, and that of PLCD3 protein was $\sim 50-60 \%$.

5-8F cell proliferation is inhibited by PLCD3 silencing. MTT assay results showed that the proliferation rate of the $5-8 \mathrm{~F}$ cells transfected with si-1 and si-2 was significantly decreased compared with the control group (Fig. 4). The results demonstrated that downregulation of PLCD3 inhibited the proliferation of 5-8F cells. 

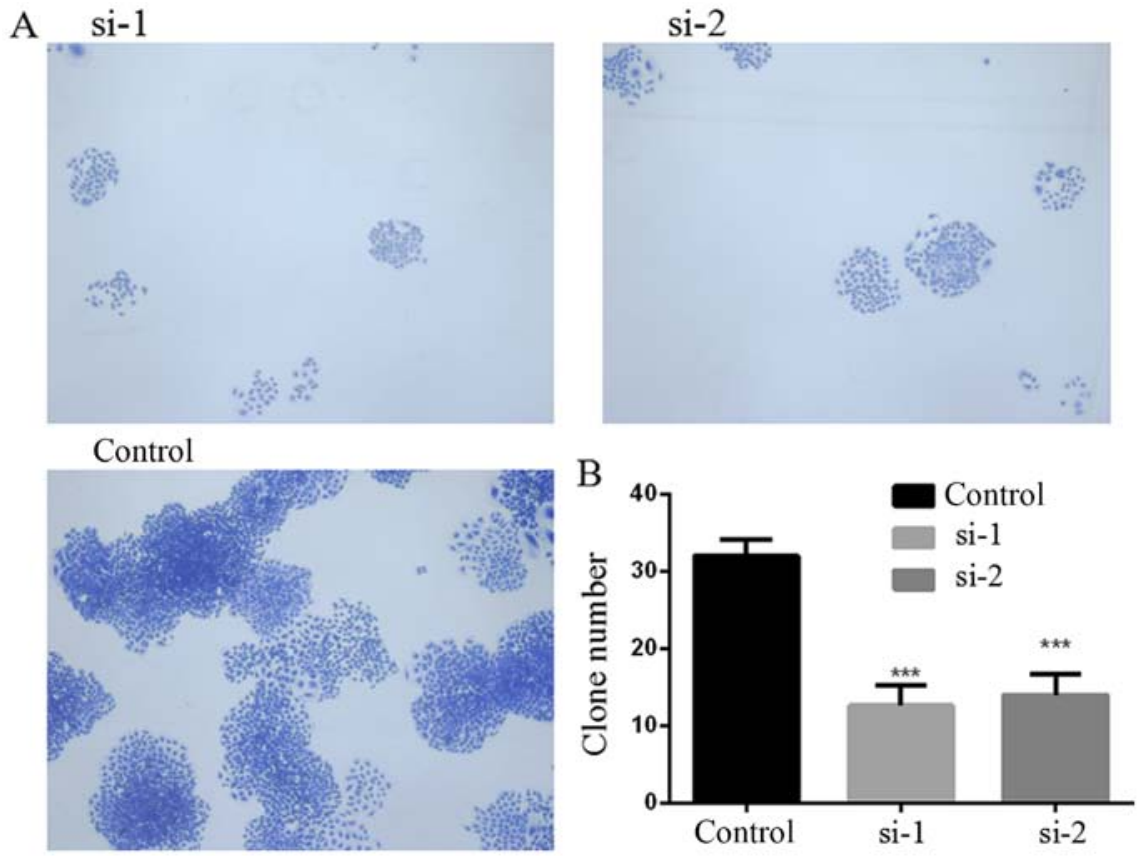

Figure 5. Effect of PLCD3 downregulation on 5-8F cell colony formation. (A) Colonies formed in 5-8F cells after transfection with PLCD3 siRNA or control RNA. (B) Colony numbers were analysed by statistical software; ${ }^{* * *} \mathrm{P}<0.001$ compared with the control group.
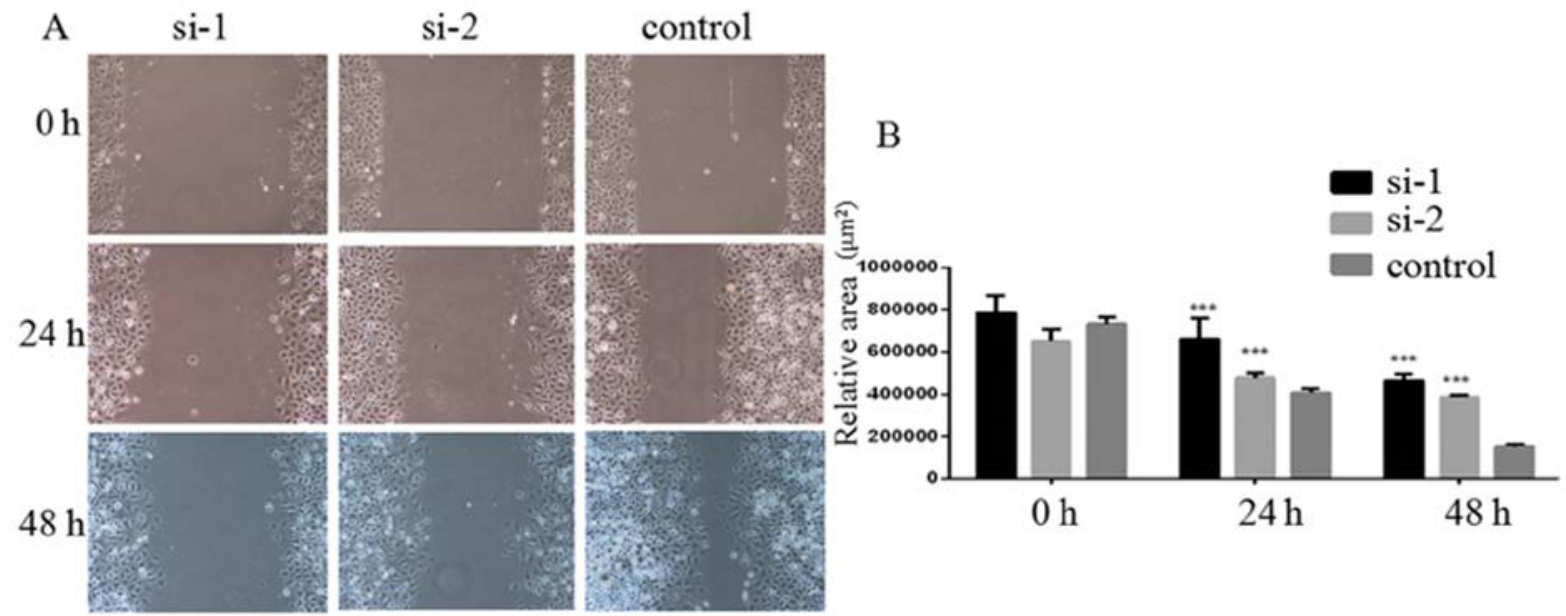

Figure 6. Effect of PLCD3 downregulation on 5-8F cell migration. (A) Migration of 5-8F cells was measured 24 and $48 \mathrm{~h}$ after transfection with PLCD3 siRNA or control RNA. (B) Migration (relative blank area) was analysed by statistical software; ${ }^{* * *} \mathrm{P}<0.001$ compared with the control group.

PLCD3 silencing inhibits the colony formation ability of 5-8F cells. The colony formation ability of 5-8F cells after transfection with PLCD3 siRNA was detected by colony formation assay. Six days after transfection with PLCD3 siRNA, as shown in Fig. 5, the number of colonies that formed in the si-1 and si- 2 groups were significantly reduced compared with this number noted in the control group.

PLCD3 silencing suppresses 5-8F cell migration. The migration of 5-8F cells after transfection with PLCD3 siRNA was detected by wound healing assays. Twenty-four to forty-eight hours after treatment, the migration was measured. As shown in Fig. 6A, the relative blank areas in the si-1 and si-2 groups were larger compared with the control group. Statistical analysis showed that transfection with si-1 and si-2 significantly inhibited 5-8F cell migration (Fig. 6B)
PLCD3 silencing impairs the invasive ability of 5-8F cells. The invasive ability of 5-8F cells after transfection with siRNA was detected by Matrigel invasion assay. Forty-eight hours after treatment, the number of cells passing through the Matrigel was counted. As shown in Fig. 7, the number of cells passing through the Matrigel in the si-1 and si-2 groups was obviously decreased compared with the control group. Statistical analysis revealed that transfection with si-1 and si-2 significantly inhibited the invasive ability of the 5-8F cells (Fig. 7B).

PLCD3 silencing influences the cell cycle of 5-8F cells. The cell cycle of $5-8 \mathrm{~F}$ cells after transfection with siRNA was detected by FACS (Fig. 8A). Compared with the control group, the number of cells in the G2/M phase was increased significantly, and those in S and G1 phase were decreased in the si-1 group. Compared with the control group, the number 

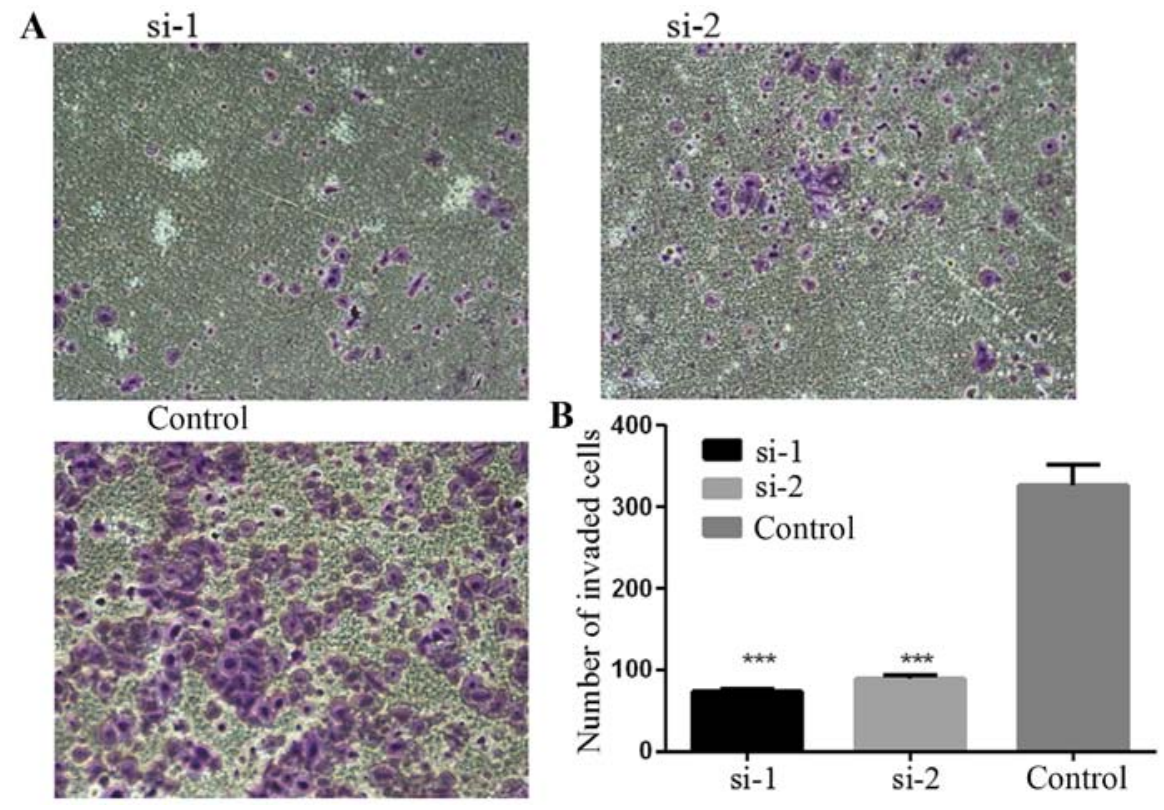

Figure 7. Effect of PLCD3 downregulation on 5-8F cell invasion. (A) Number of invaded cells was counted $48 \mathrm{~h}$ after transfection with PLCD3 siRNA or control RNA. (B) Number of invaded cells was analysed by statistical software; ${ }^{* * * *} \mathrm{P}<0.001$ compared with the control group.
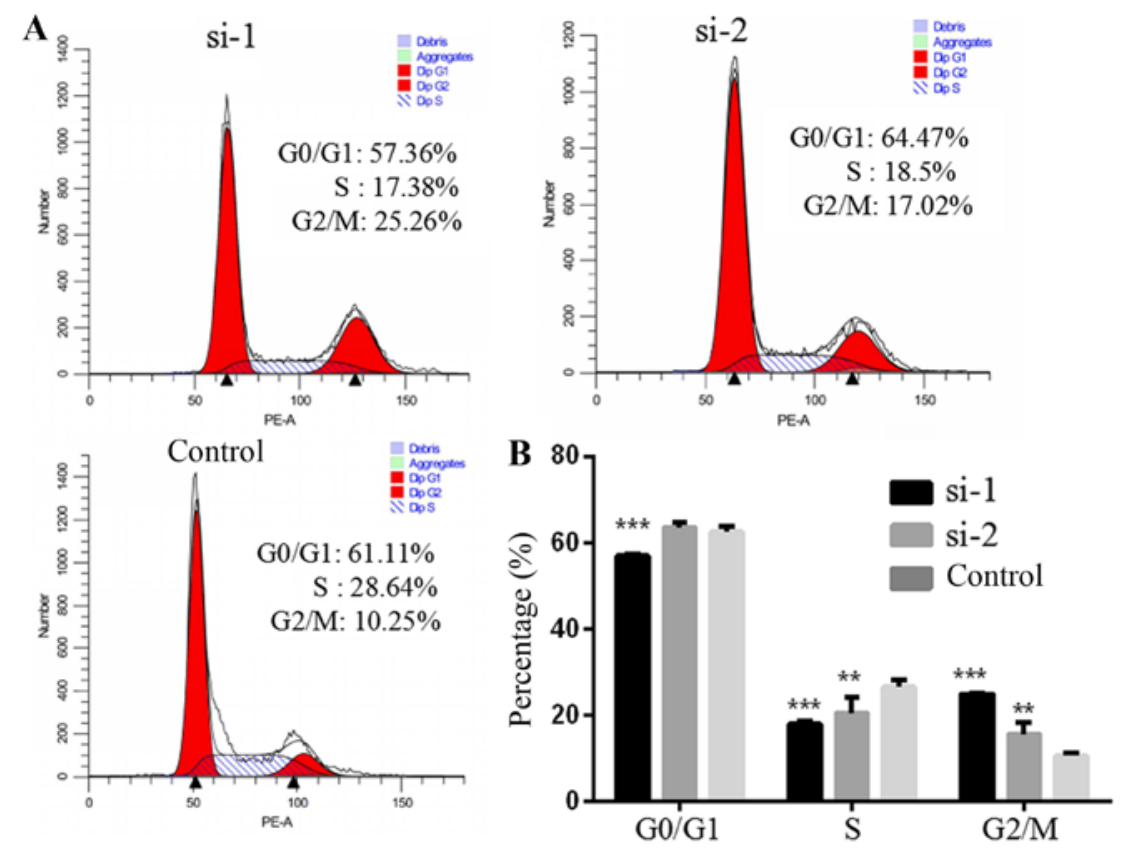

Figure 8. Effect of PLCD3 downregulation on the cell cycle distribution in 5-8F cells. (A) The effect of PLCD3 knockdown on the cell cycle in 5-8F cells was analysed by flow cytometry. (B) The proportion of cells in each cell cycle was analysed by statistical software; ${ }^{* * *} \mathrm{P}<0.001,{ }^{* *} \mathrm{P}<0.01$ compared with the control group, in which 5-8F cells were transfected with control siRNA.

of cells in the G2/M phase was significantly increased. The number of $\mathrm{S}$ phase cells was decreased, and the number of G1 phase cells was not significantly altered in the si-2 group (Fig. 8B).

PLCD3 silencing influences the expression of Flot2 and several proteins related to EMT and invasion. We found that the invasion and migration ability of the $5-8 \mathrm{~F}$ cells was inhibited after silencing of PLCD3, thus we detected the expression of several proteins related to EMT and invasion including MMP2, MMP9, Snai1, E-cadherin, N-cadherin and vimentin. At the same time, we detected the expression of the interaction protein Flot2. After silencing the expression of PLCD3, the expression levels of MMP2, MMP9, Snai1, vimentin, $\mathrm{N}$-cadherin were decreased, and the expression level of E-cadherin was increased. However, the expression of Flot2 was not significantly altered (Fig. 9).

\section{Discussion}

In the present study, we found that PLCD3 interacts with Flot2. Flot2 is highly expressed and associated with tumour 


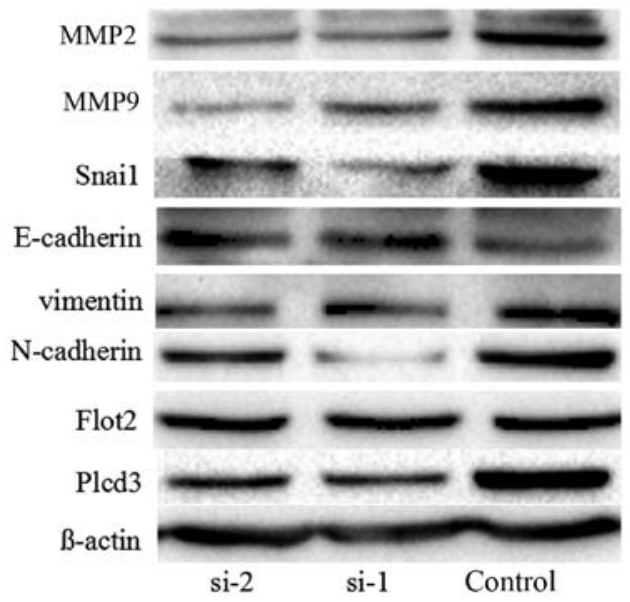

Figure 9. Effect of PLCD3 downregulation on the expression of Flot2 and several proteins related to EMT and invasion were detected by western blotting. Silencing of PLCD3 expression decreased the expression of MMP2, MMP9, Snai1, vimentin and N-cadherin, and increased E-cadherin expression. However, the expression of Flot 2 was not significantly influenced.

progression and metastasis in a variety of tumours $(8,44-53)$, suggesting that it may be an independent diagnostic marker and potential therapeutic target. Our previous research revealed that downregulation of Flot 2 in 5-8F cells led to reductions in colony formation, proliferation, migration and invasion; and inhibited cell cycle progression. In addition, it is associated with NPC metastasis (8).

Phosphoinositide-specific phospholipase C (PLC) catalyses phosphatidylinositol 4,5-bisphosphate (PIP2) into two second messengers, which play important roles in cell movement, growth and diseases. To date, the roles of PLC in the progression of cancers have rarely been investigated. PLC- $\gamma 1$ may play a role in cancer cell invasion, metastasis and breast tumour formation $(27,28)$. PLC- $\delta 1$ and PLC- $\varepsilon$ are regarded as tumour suppressors (32-35), whereas PLC- $\beta 3$ is associated with lymphomas and other tumours, such as myeloid malignancies (37). PLCD3 plays important roles in numerous biological processes, such as survival of cardiomyocytes and trophoblasts, maintaining normal heart function and promoting neurite expansion (54-56). Studies concerning the roles of PLCD3 in tumours are rare. To the best of our knowledge, there is only one study demonstrating that PLCD3 can promote the proliferation and migration of neoplastic mammary epithelial cells (57). The role of PLCD3 in NPC has not been studied to date. Thus, we ascertained whether PLCD3 plays a certain role in NPC. In the present study, PLCD3 silencing in 5-8F cells by siRNA inhibited colony formation, proliferation, migration and invasion and blocked cell cycle progression. These results are consistent with previous findings.

We also analysed the effects of PLCD3 silencing on the expression of several proteins related to EMT and invasion including MMP2, MMP9, Snai1, E-cadherin, N-cadherin and vimentin. After silencing the expression of PLCD3, the expression levels of MMP2, MMP9, Snai1, vimentin and $\mathrm{N}$-cadherin were apparently decreased, and the expression level of E-cadherin was apparently increased, which is in accordance with our previous study on Flot2 (8).

In conclusion, we first discovered the interaction between Flot2 and PLCD3 using the Y2H system and verified this interaction by co-immunoprecipitation. The roles of PLCD3 in promoting proliferation, migration, and invasion of NPC cells were also demonstrated by silencing its expression with siRNAs. Flot2 plays an important role in the progression of NPC, which may be partially related to its interaction with PLCD3.

\section{Acknowledgements}

The present study was supported by the National Natural Science Foundation of China (nos. 81773179 and 81272972), the Natural Science Foundation of Hunan Province of China (nos. 2016JJ2172), Mittal Student Innovation Project, the National Basic Research Program of China (2010CB833605), the Hunan Provincial Science and Technology Department (nos. 2016JC2049, 2014FJ6006 and 2013FJ4010), and the Open-End Fund for the Valuable and Precision Instruments of Central South University.

\section{References}

1. Sham JS, Wei WI, Zong YS, Choy D, Guo YQ, Luo Y, Lin ZX and $\mathrm{Ng} \mathrm{MH:} \mathrm{Detection} \mathrm{of} \mathrm{subclinical} \mathrm{nasopharyngeal} \mathrm{carcinoma} \mathrm{by}$ fibreoptic endoscopy and multiple biopsy. Lancet 335: 371-374, 1990.

2. Feng X, Ren C, Zhou W, Liu W, Zeng L, Li G, Wang L, Li M, Zhu B, Yao K, et al: Promoter hypermethylation along with $\mathrm{LOH}$, but not mutation, contributes to inactivation of $D L C-1$ in nasopharyngeal carcinoma. Mol Carcinog 53: 858-870, 2014.

3. Zhang L, Chen QY, Liu H, Tang LQ and Mai HQ: Emerging treatment options for nasopharyngeal carcinoma. Drug Des Devel Ther 7: 37-52, 2013.

4. Brückner A, Polge C, Lentze N, Auerbach D and Schlattner U: Yeast two-hybrid, a powerful tool for systems biology. Int J Mol Sci 10: 2763-2788, 2009.

5. James P, Halladay J and Craig EA: Genomic libraries and a host strain designed for highly efficient two-hybrid selection in yeast. Genetics 144: 1425-1436, 1996.

6. Yang XY, Ren CP, Wang L, Li H, Jiang CJ, Zhang HB, Zhao M and Yao KT: Identification of differentially expressed genes in metastatic and non-metastatic nasopharyngeal carcinoma cells by suppression subtractive hybridization. Cell Oncol 27: 215-223, 2005.

7. Wen Q, Li J, Wang W, Xie G, Xu L, Luo J, Chu S, She L, Li D, Huang D, et al: Increased expression of flotillin-2 protein as a novel biomarker for lymph node metastasis in nasopharyngeal carcinoma. PLoS One 9: e101676, 2014.

8. Liu J, Huang W, Ren C, Wen Q, Liu W, Yang X, Wang L, Zhu B, Zeng L, Feng X, et al: Flotillin-2 promotes metastasis of nasopharyngeal carcinoma by activating NF- $\kappa \mathrm{B}$ and PI3K/Akt3 signaling pathways. Sci Rep 5: 11614, 2015.

9. Cao K, Xie D, Cao P, Zou Q, Lu C, Xiao S, Zhou J and Peng X: SiRNA-mediated flotillin-2 (Flot2) downregulation inhibits cell proliferation, migration, and invasion in gastric carcinoma cells. Oncol Res 21: 271-279, 2014.

10. Nishizuka Y: Protein kinase $\mathrm{C}$ and lipid signaling for sustained cellular responses. FASEB J 9: 484-496, 1995.

11. De Smedt H and Parys JB: Molecular and functional diversity of inositol triphosphate-induced $\mathrm{Ca}^{2+}$ release. Verh $\mathrm{K}$ Acad Geneeskd Belg 57: 423-458, 1995 (In Dutch).

12. Berridge MJ: Inositol trisphosphate and calcium signalling. Nature 361: 315-325, 1993.

13. Cockcroft $\mathrm{S}$ and Thomas GM: Inositol-lipid-specific phospholipase $\mathrm{C}$ isoenzymes and their differential regulation by receptors. Biochem J 288: 1-14, 1992.

14. Rhee SG and Bae YS: Regulation of phosphoinositide-specific phospholipase C isozymes. J Biol Chem 272: 15045-15048, 1997.

15. Kelley GG, Reks SE, Ondrako JM and Smrcka AV: Phospholipase C(epsilon): A novel Ras effector. EMBO J 20: 743-754, 2001.

16. Harden TK and Sondek J: Regulation of phospholipase C isozymes by ras superfamily GTPases. Annu Rev Pharmacol Toxicol 46: 355-379, 2006.

17. Katan M and Williams RL: Phosphoinositide-specific phospholipase C: Structural basis for catalysis and regulatory interactions. Semin Cell Dev Biol 8: 287-296, 1997.

18. Rhee SG: Reflections on the days of phospholipase C. Adv Biol Regul 53: 223-231, 2013. 
19. García del Caño G, Montaña M, Aretxabala X, GonzálezBurguera I, López de Jesús M, Barrondo S and Sallés J: Nuclear phospholipase C- $\beta 1$ and diacylglycerol LIPASE- $\alpha$ in brain cortical neurons. Adv Biol Regul 54: 12-23, 2014.

20. Koh HY: Phospholipase C- $\beta 1$ and schizophrenia-related behaviors. Adv Biol Regul 53: 242-248, 2013.

21. Follo MY, Faenza I, Fiume R, Ramazzotti G, McCubrey JA, Martelli AM, Manzoli FA and Cocco L: Revisiting nuclear phospholipase C signalling in MDS. Adv Biol Regul 52: 2-6, 2012.

22. Follo MY, Marmiroli S, Faenza I, Fiume R, Ramazzotti G, Martelli AM, Gobbi P, McCubrey JA, Finelli C, Manzoli FA, et al: Nuclear phospholipase $C \beta 1$ signaling, epigenetics and treatments in MDS. Adv Biol Regul 53: 2-7, 2013.

23. Manzoli L, Mongiorgi S, Clissa C, Finelli C, Billi AM, Poli A, Quaranta M, Cocco L and Follo MY: Strategic role of nuclear inositide signalling in myelodysplastic syndromes therapy. Mini Rev Med Chem 14: 873-883, 2014.

24. Zaidi SK, Trombly DJ, Dowdy CR, Lian JB, Stein JL, van Wijnen AJ and Stein GS: Epigenetic mechanisms in leukemia. Adv Biol Regul 52: 369-376, 2012.

25. Barker CJ, Li L, Köhler M and Berggren PO: $\beta$-Cell $\mathrm{Ca}^{2+}$ dynamics and function are compromised in aging. Adv Biol Regul 57: 112-119, 2015.

26. Jang HJ, Yang YR, Kim JK, Choi JH, Seo YK, Lee YH, Lee JE, Ryu SH and Suh PG: Phospholipase C- $\gamma 1$ involved in brain disorders. Adv Biol Regul 53: 51-62, 2013.

27. Lattanzio R, Piantelli M and Falasca M: Role of phospholipase $C$ in cell invasion and metastasis. Adv Biol Regul 53: 309-318, 2013.

28. Arteaga CL, Johnson MD, Todderud G, Coffey RJ, Carpenter G and Page DL: Elevated content of the tyrosine kinase substrate phospholipase C-gamma 1 in primary human breast carcinomas. Proc Natl Acad Sci USA 88: 10435-10439, 1991.

29. Nakamura Y, Kanemarum K and Fukami K: Physiological functions of phospholipase C 81 and phospholipase C83. Adv Biol Regul 53: 356-362, 2013.

30. Shimohama S, Perry G, Richey P, Takenawa T, Whitehouse PJ, Miyoshi K, Suenaga T, Matsumoto S, Nishimura $M$ and Kimura J: Abnormal accumulation of phospholipase C-delta in filamentous inclusions of human neurodegenerative diseases. Neurosci Lett 162: 183-186, 1993.

31. Shimohama S, Homma Y, Suenaga T, Fujimoto S, Taniguchi T, Araki W, Yamaoka Y, Takenawa T and Kimura J: Aberrant accumulation of phospholipase C-delta in Alzheimer brains. Am J Pathol 139: 737-742, 1991.

32. Fu L, Qin YR, Xie D, Hu L, Kwong DL, Srivastava G, Tsao SW and Guan XY: Characterization of a novel tumor-suppressor gene PLC delta 1 at 3p22 in esophageal squamous cell carcinoma. Cancer Res 67: 10720-10726, 2007.

33. Hu XT, Zhang FB, Fan YC, Shu XS, Wong AH, Zhou W, Shi QL, Tang HM, Fu L, Guan XY, et al: Phospholipase C delta 1 is a novel $3 \mathrm{p} 22.3$ tumor suppressor involved in cytoskeleton organization, with its epigenetic silencing correlated with high-stage gastric cancer. Oncogene 28: 2466-2475, 2009.

34. Chan JJ and Katan M: PLC $\varepsilon$ and the RASSF family in tumour suppression and other functions. Adv Biol Regul 53: 258-279, 2013.

35. Wang X, Zbou C, Qiu G, Fan J, Tang H and Peng Z: Screening of new tumor suppressor genes in sporadic colorectal cancer patients. Hepatogastroenterology 55: 2039-2044, 2008.

36. Amdani SN, Jones C and Coward K: Phospholipase C zeta (PLC $\zeta$ ): Oocyte activation and clinical links to male factor infertility. Adv Biol Regul 53: 292-308, 2013.

37. Xiao W, Hong H, Kawakami Y, Kato Y, Wu D, Yasudo H, Kimura A, Kubagawa H, Bertoli LF, Davis RS, et al: Tumor suppression by phospholipase C-beta3 via SHP-1-mediated dephosphorylation of Stat5. Cancer Cell 16: 161-171, 2009.

38. Shi J, Ren C, Liu H, Wang L, Zhu B, Huang W, Liu W, Liu J, Liu Y, $\mathrm{Xia} X$, et al: An ESRG-interacting protein, COXII, is involved in pro-apoptosis of human embryonic stem cells. Biochem Biophys Res Commun 460: 130-135, 2015.

39. Wanggou S, Jiang X, Li Q, Zhang L, Liu D, Li G, Feng X, Liu W, Zhu B, Huang W, et al: HESRG: A novel biomarker for intracranial germinoma and embryonal carcinoma. J Neurooncol 106: $251-259,2012$.
40. Tang G, Liu D, Xiao G, Liu Q and Yuan J: Transcriptional repression of FOXO1 by KLF4 contributes to glioma progression. Oncotarget 7: 81757-81767, 2016.

41. Zhou W, Feng X, Ren C, Jiang X, Liu W, Huang W, Liu Z, Li Z, Zeng L, Wang L, et al: Over-expression of $B C A T 1$, a c-Myc target gene, induces cell proliferation, migration and invasion in nasopharyngeal carcinoma. Mol Cancer 12: 53, 2013.

42. Zhang H, Feng X, Liu W, Jiang X, Shan W, Huang C, Yi H, Zhu B, Zhou W, Wang L, et al: Underlying mechanisms for LTF inactivation and its functional analysis in nasopharyngeal carcinoma cell lines. J Cell Biochem 112: 1832-1843, 2011.

43. Feng X, Li C, Liu W, Chen H, Zhou W, Wang L, Zhu B, Yao K, Jiang $\mathrm{X}$ and Ren C: $D L C-1$, a candidate tumor suppressor gene, inhibits the proliferation, migration and tumorigenicity of human nasopharyngeal carcinoma cells. Int J Oncol 42: 1973-1984, 2013.

44. Hazarika P, McCarty MF, Prieto VG, George S, Babu D, Koul D, Bar-Eli M and Duvic M: Up-regulation of Flotillin-2 is associated with melanoma progression and modulates expression of the thrombin receptor protease activated receptor 1 . Cancer Res 64: 7361-7369, 2004.

45. Gómez V, Sesé M, Santamaría A, Martínez JD, Castellanos E, Soler M, Thomson TM and Paciucci R: Regulation of aurora B kinase by the lipid raft protein flotillin-1. J Biol Chem 285: 20683-20690, 2010.

46. Satyamoorthy K, Li G, Gerrero MR, Brose MS, Volpe P, Weber BL, Van Belle P, Elder DE and Herlyn M: Constitutive mitogen-activated protein kinase activation in melanoma is mediated by both BRAF mutations and autocrine growth factor stimulation. Cancer Res 63: 756-759, 2003.

47. Liu Y, Lin L, Huang Z, Ji B, Mei S, Lin Y and Shen Z: High expression of flotillin-2 is associated with poor clinical survival in cervical carcinoma. Int J Clin Exp Pathol 8: 622-628, 2015.

48. Berger T, Ueda T, Arpaia E, Chio II, Shirdel EA, Jurisica I, Hamada K, You-Ten A, Haight J, Wakeham A, et al: Flotillin-2 deficiency leads to reduced lung metastases in a mouse breast cancer model. Oncogene 32: 4989-4994, 2013.

49. Wang YL, Yao WJ, Guo L, Xi HF, Li SY and Wang ZM: Expression of flotillin-2 in human non-small cell lung cancer and its correlation with tumor progression and patient survival. Int J Clin Exp Pathol 8: 601-607, 2015.

50. Lin C, Wu Z, Lin X, Yu C, Shi T, Zeng Y, Wang X, Li J and Song L: Knockdown of FLOT1 impairs cell proliferation and tumorigenicity in breast cancer through upregulation of FOXO3a. Clin Cancer Res 17: 3089-3099, 2011.

51. Yan Y, Yang FQ, Zhang HM, Che J and Zheng JH: Up-regulation of flotillin-2 is associated with renal cell carcinoma progression. Tumour Biol 35: 10479-10486, 2014.

52. Zhao L, Lin L, Pan C, Shi M, Liao Y, Bin J and Liao W: Flotillin-2 promotes nasopharyngeal carcinoma metastasis and is necessary for the epithelial-mesenchymal transition induced by transforming growth factor- $\beta$. Oncotarget 6: 9781-9793, 2015.

53. Takano N, Iizuka N, Hazama S, Yoshino S, Tangoku A and Oka M: Expression of estrogen receptor-alpha and -beta mRNAs in human gastric cancer. Cancer Lett 176: 129-135, 2002.

54. Nakamura Y, Hamada Y, Fujiwara T, Enomoto H, Hiroe T, Tanaka S, Nose M, Nakahara M, Yoshida N, Takenawa T, et al: Phospholipase C-delta1 and -delta3 are essential in the trophoblast for placental development. Mol Cell Biol 25: 10979-10988, 2005.

55. Nakamura Y, Kanemaru K, Kojima R, Hashimoto Y, Marunouchi T, Oka N, Ogura T, Tanonaka K and Fukami K: Simultaneous loss of phospholipase $\mathrm{C} \delta 1$ and phospholipase $\mathrm{C} \delta 3$ causes cardiomyocyte apoptosis and cardiomyopathy. Cell Death Dis 5: e1215, 2014

56. Kouchi Z, Igarashi T, Shibayama N, Inanobe S, Sakurai K, Yamaguchi H, Fukuda T, Yanagi S, Nakamura Y and Fukami K: Phospholipase Cdelta3 regulates RhoA/Rho kinase signaling and neurite outgrowth. J Biol Chem 286: 8459-8471, 2011.

57. Rebecchi MJ, Raghubir A, Scarlata S, Hartenstine MJ, Brown T and Stallings JD: Expression and function of phospholipase $\mathrm{C}$ in breast carcinoma. Adv Enzyme Regul 49: 59-73, 2009.

This work is licensed under a Creative Commons Attribution-NonCommercial-NoDerivatives 4.0 International (CC BY-NC-ND 4.0) License. 